\title{
The Physics of Ice
}

\section{Yu.A. Ossipyan and V.F. Petrenko, Chernogolovka}

\author{
(Institute of Solid State Physics, USSR Academy of Sciences)
}

\begin{abstract}
Recent studies on ice carried out in the Institute of Solid State Physics of the USSR Academy of Sciences suggest that traditional concepts need revision.
\end{abstract}

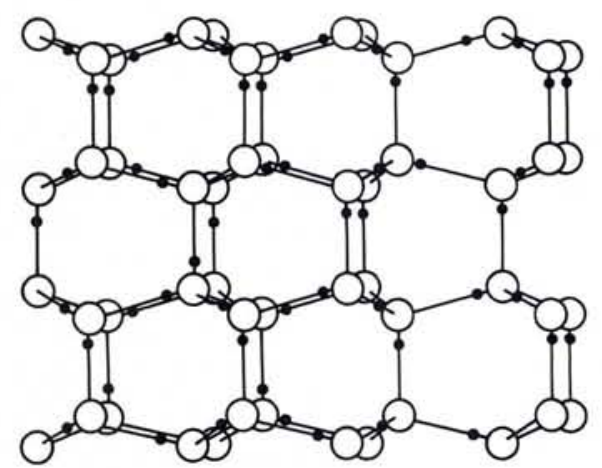

Fig. 1 - A typical disordered arrangement of protons in an ice structure. Each oxygen has two close protons, forming an $\mathrm{H}_{2} \mathrm{O}$ molecule, and there is one proton on each bond.

Ice has not been a traditional topic for physics research in the past, so an introduction to the background to our investigations would not be out of place. Ice and snow play an important role both in the life of individuals and for our planet as a whole. Vast masses of ice, stored in kilometer-thick caps in Antarctica and Greenland, in rock glaciers and as covers to the polar seas, in permafrost layers and seasonal snow covers, determine to a great extent the amount of solar energy absorbed by the Earth, as well as heat and water transfer in the atmosphere, and many other factors contributing to the ecological equilibrium.

Recently, ice science has become important in diverse fields of scientific, social and industrial activity, such as climatology, astrophysics, meteorology, navigation, building in cold regions, medicine and biology, functioning of technical equipment, production and transportation of oil and gas, defence, geology, exploitation of pavements etc.

The basis of the science of ice and snow was laid by such physicists as Faraday and Laue, the Nobel laureates Pauling, Onsager and Eigen, and many others. Through their efforts, by the beginning of the 1970s the essential principles of modern ice physics had been formulated. Their investigations had revealed a number of unique properties, making ice one of the most interesting objects of research in solid state physics.
Compared with other solids, ice is distinct in its structure, since it consists of a crystalline lattice of oxygen atoms and a disordered proton subsystem, whose configurations constantly vary (Fig. 1). Nevertheless, for all configurations, each oxygen atom retains two protons, thus conserving the chemical formula $\mathrm{H}_{2} \mathrm{O}$. The transition from one configuration to any other may be regarded as a rotation of the water molecules and under typical conditions $\left(T=-10^{\circ} \mathrm{C}\right)$ each water molecule in the ice crystal can turn at an angular rate $\omega \cong 10^{6} \mathrm{~s}^{-1}$. Moreover, if the number of water molecules in the ice lattice equals $N$, the total number of possible configurations is close to $(3 / 2)^{N}$. Because of the isoenergetic character of all these configurations, the configurational entropy (zeropoint entropy) $S=k_{\mathrm{B}} N \ln (3 / 2)$ is significant and does not disappear as $T \rightarrow 0$, seemingly breaching the third law of thermodynamics.

A number of basic properties of ice are related to this ability of water molecules to reorientate. For example, as the molecules have a considerable electric dipole moment, the static dielectric permittivity of ice is large $\left(\varepsilon_{\mathrm{S}} \approx 100\right)$. Reorientation also accounts for the additional (over and above elastic) deformation of ice under the action of mechanical stresses - the so-called inelastic relaxation of ice.

To arrive at a real mechanism by which this reorientation can happen in the ice lattice, four types of defect of the proton subsystem have been introduced: $\mathrm{H}_{3} \mathrm{O}^{+}$ and $\mathrm{OH}^{-}$ions (Figs. 2, 3) and L- and Ddefects. Their motion through the ice results in the molecules turning and in electric charge transfer. Interestingly, this charge is fractional $[1,2]$ :

$$
\begin{gathered}
e_{\mathrm{H}_{3} \mathrm{O}^{+}}=0.62 \mathrm{e} ; e_{\mathrm{D}}=0.38 \mathrm{e} ; \\
e_{\mathrm{H}_{3} \mathrm{O}^{+}}+e_{\mathrm{D}}=\mathrm{e}
\end{gathered}
$$

The effective charge transported by an ionic defect is less than the protonic one since the jump of the defect from one water molecule to the next corresponds to the real displacement of a proton over a distance less than the intermolecular space (Fig. 2b). The same is valid for L- and D-defects (Fig. 3b).
One should bear in mind that in the motion of defects, protons alone are actually moving, and it is protons that are the principal current (charge) carriers. Electron conduction does not play any important role owing to the large width of the gap $E_{\mathrm{G}} \cong 10.9 \mathrm{eV}$. In contrast, the activation energies of the formation of a pair of ions $E_{\mathrm{i}} \cong 0.96 \mathrm{eV}$ and a pair of Bjerrum (L, D) defects $E_{\mathrm{o}} \cong 0.68 \mathrm{eV}$ are close to the width of the energy gap $E_{\mathrm{G}}$ in normal semiconductors, so that in regard to carrier concentration and absolute value of conduction, ice can be ranked as a proton semiconductor.

These principal features of ice, briefly described here, explained so many of its physical properties that in the early 1970 s it was believed that we had evolved a nearly complete and unambiguous picture. However, investigations that have been carried out in the Institute of Solid State Physics of the USSR Academy of Sciences since the beginning of the 1980 s with the aim of closing small "white spots" on the physical map of ice, have revealed a number of new physical phenomena. That, in turn, has given rise to serious doubts concerning the validity of existing conceptions.
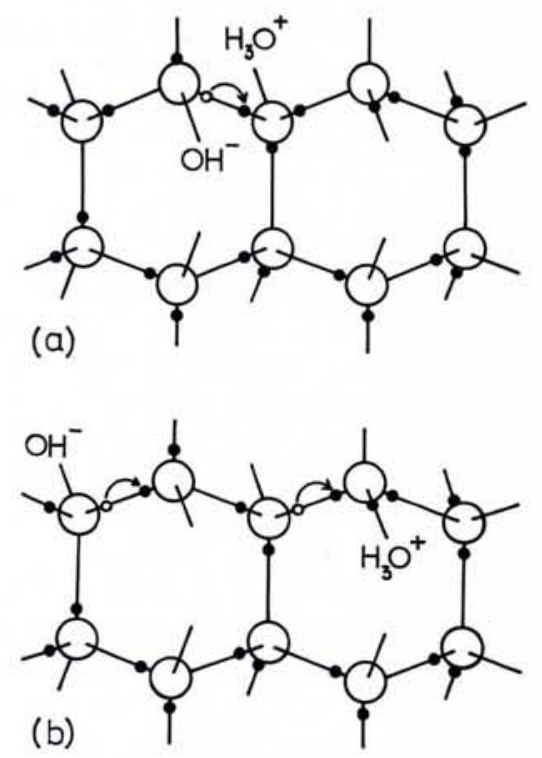

Fig. 2 - a) Formation of an ion pair, $\mathrm{H}_{3} \mathrm{O}$ and $\mathrm{OH}^{-}$, by a proton jump along a bond. b) Migration of these two ions by further proton jumps. 

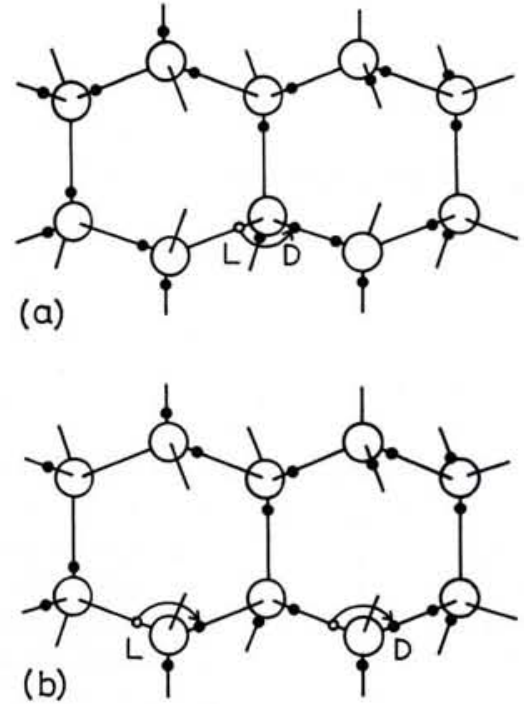

Fig. 3 - a) Formation of an orientational defect pair, $D$ - and $L$-, by an oblique proton jump.

b) Migration of these two L- and D-defects by further oblique proton jumps.

\section{Nature of Current Carriers in Ice}

Our notions on the proton electric conduction of ice were based, first on measurements of hydrogen release at the cathode in the presence of direct currents, carried out in the 1950s. Second on the model of Jaccard [1], who developed a very elegant method based on the co-ordinated motion of the two types of ions and D- and L-defects. It is clear now that the verification of Faraday's law, carried out in the 1950s, does not answer the question about the nature of the charge carriers, since in the experimental conditions obtaining, the currents flowed almost totally across the quasi-liquid surface layer rather than across the bulk. These rather complicated and labour-consuming experiments will have to be repeated. Moreover, some experimental results recently obtained by us, do not fit Jaccard's model.

First of all, direct currents flowing through the ice between metallic electrodes were found to remain invariable over days. The great amounts of hydrogen and oxygen, that should have been released at the contacts according to Faraday's law, should have disturbed the continuity, but this was not the case. It then turned out that the DC flowing through the interface between the electronic conductor and the ice was very sensitive to the difference between the electron work functions of the ice and the electrode. For example, Fig. 4 shows the effect of illumination on the current through the interface using a common semiconductor ( $\mathrm{CdSe}$ ) as electrode. The light passes through the ice and is absorbed in a thin ( $\cong 1 \mu \mathrm{m}$ ) layer, altering appreciably the Fermi level position in the CdSe [3]. In another series of experiments, we succeeded in increasing the electric conduction of the ice-metal interface by two orders of magnitude, by forming double electric layers at the interface, and aligning the Fermi level in the metal with the position of the electronic level of $\mathrm{OH}^{-}$ions in the ice (Fig. 5).

These experiments show that the electron exchange between the ice and the electronic conductors has a direct impact on the electric conduction of the ice. However, the life time of free electrons in ice $\left(10^{-9}-10^{-10} \mathrm{~s}\right)$ is insignificantly small and their mobility is low, which excludes "purely electronic" conduction. At the same time, it was found that on injecting protons into ice from hydrogen-saturated electrodes, an increase of both static and high frequency electric conduction resulted [4].

Notwithstanding the fact that quantitatively these experiments cannot be explained in terms of Jaccard's model, they unambiguously indicate the participation of protons in the processes of electric conduction. In the light of this, we have devised a model of combined conduction [3] according to which, ions $\mathrm{H}_{3} \mathrm{O}^{+}$and $\mathrm{OH}^{-}$arrive at the electrodes and exchange electrons with them, but because of their fractional charge $( \pm$ $0.62 \mathrm{e}$ ) do not become neutral (as they would in water). Instead, they acquire charges $(\mp 0.38 \mathrm{e}$ ) and become charged "radicals" $\left(\mathrm{H}_{3} \mathrm{O}\right)^{\circ}$. After that they start moving towards the opposite electrodes where they again become $\mathrm{H}_{3} \mathrm{O}^{+}$and $\mathrm{OH}^{-}$ions. It should be noted that the fractional charge of the "radicals" arises in the same way as for ions, because the proton displacement is less than an intermolecular distance, when a "radical" displaces from one molecule to another. At the same time, the electron trapped

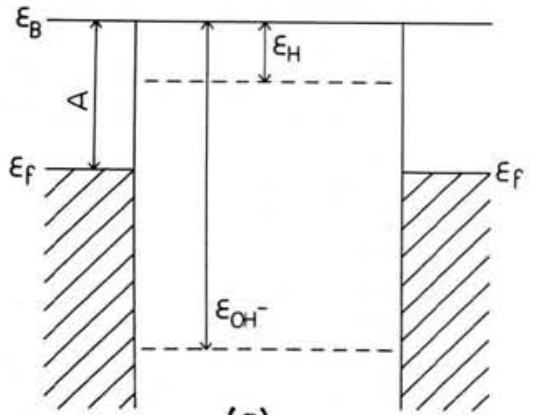

(a)

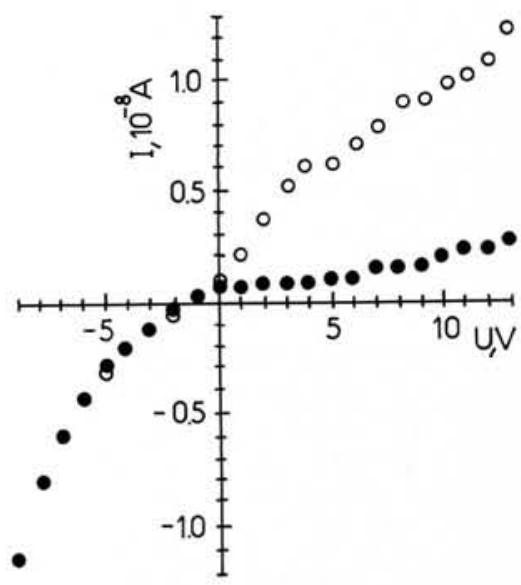

Fig. 4-Current-voltage characteristics of the CdSe-ice system under illumination lopen circles) and in the dark (He-Ne laser, $T=-10^{\circ} \mathrm{C}$.

by a defect covers the whole distance, and the following relation should be fulfilled:

$$
\begin{aligned}
& e_{\mathrm{H}^{*}}=e_{\mathrm{H}_{3} \mathrm{O}^{+}}-\mathrm{e}=e_{\mathrm{L}}=-0.38 \mathrm{e} \\
& e_{\mathrm{OH}^{*}}=e_{\mathrm{OH}^{-}}+\mathrm{e}=e_{\mathrm{D}}=+0.38 \mathrm{e}
\end{aligned}
$$

Not only the absolute values of charges of $\mathrm{H}^{\bullet}$ and $\mathrm{OH}^{*}$ coincide with the charges of $\mathrm{L}$ - and $\mathrm{D}$-defects. The reorientations of molecules of water along the course of motion of an L-defect and $\mathrm{H}^{\text {* "radical" }}$ and of a D-defect and $\mathrm{OH}^{\text {" "radical" are }}$ the same. This allows $\mathrm{H}^{\bullet}$ and $\mathrm{OH}^{\bullet}$ "radicals" to be substituted for L- and D-defects in the Jaccard model in such a way that all the predictions of that model will remain unaltered.

The life times of the "radicals", owing to Coulomb repulsion are greater by several orders of magnitude than the normally small life times of electrically neutral radicals in liquids, determined by the reaction type

$$
\begin{aligned}
(\mathrm{OH})^{*}+(\mathrm{OH})^{\bullet} & \rightarrow \mathrm{H}_{2} \mathrm{O}_{2} \\
(\mathrm{H})^{\bullet}+(\mathrm{H})^{\bullet} & \rightarrow \mathrm{H}_{2}
\end{aligned}
$$

which also suggests that they par-

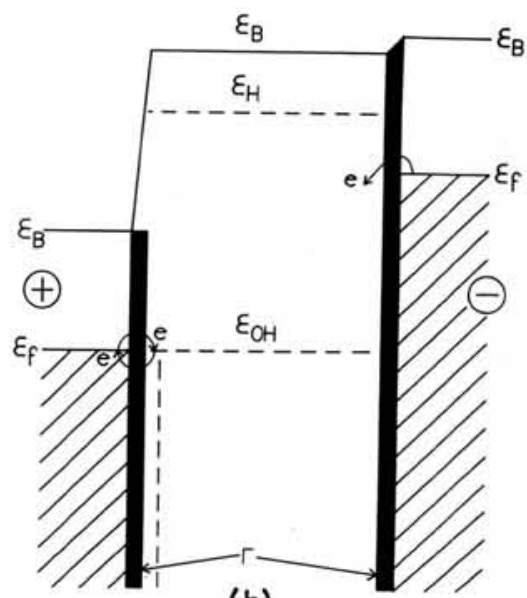

(b)

Fig. 5-Electronic energy levels of ice and metal electrodes: a) before and b) after formation of charged double layers $(\Gamma)$. $\varepsilon_{f}$-Fermi level of electrons in metal, $\varepsilon_{B}$-energy of electrons in vacuum, $\varepsilon_{H^{-}}$-work function of $\mathrm{H}$ in ice, $\varepsilon_{\mathrm{OH}^{-}}$-work function of $\mathrm{OH}$ in ice. 

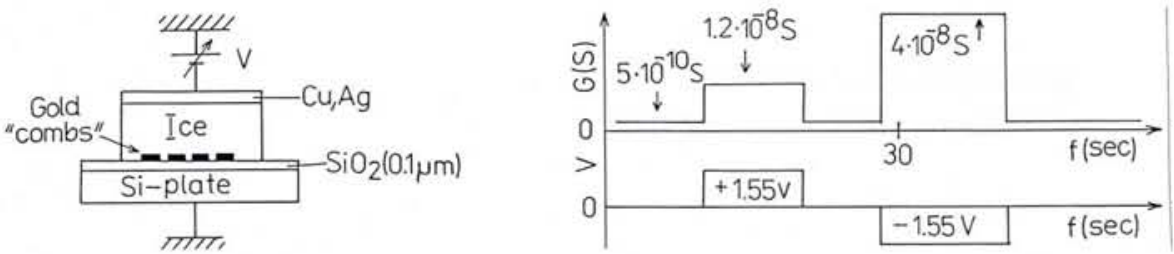

Fig. 6 - Ice field-effect transistor. (Left) Experimental set-up. (Right) Time diagram of the surface AC conductivity of ice, measured at frequency $f=10 \mathrm{~Hz}, T=-33.1^{\circ} \mathrm{C}$. The lower curve represents the potential difference between the Si plate and the ground.

ticipate in the processes of electric conduction. The model also is supported indirectly by observations on the bulk photoconductivity of ice under ultraviolet light, where exciting electronic transitions results in the appearance of $\mathrm{OH}^{\bullet}$ "radicals" [5]. Further evidence of the existence of charged "radicals" is the small amount of hydrogen peroxide, $\mathrm{H}_{2} \mathrm{O}_{2}$, observed in ice following the passage of an electric current.

For a long time, research into the nature of charge carriers in ice was hindered by the lack of convenient methods for determining their mobility $\mu$. Because $\mu$ is small, $\left(10^{-2}-10^{-4}\right) \mathrm{cm}^{2} / \mathrm{Ns}$, methods based on the measurement of the Hall effect are not applicable. However, physicists at the Institute of Solid State Physics, USSR Ac Sci together with physicists at Birmingham University, and at the Institute of Low Temperature Sciences, Hokkaido University have proposed three complementary methods for the determination of carrier mobilities in ice $[4,6,7]$. Two of these measure the electronic conduction of ice, by producing a controlled excessive carrier concentration, either by proton injection into the ice bulk [4] or by creating a field transistor in the nearsurface layer (Fig. 6a, b) [7].

With the third method, concentrations and mobilities of several types of carrier can be determined from measurements of the frequency dependence of the real part of the dielectric permittivity $\varepsilon^{\prime}$ (Fig. 7). As shown in the work by Ryzhkin and Petrenko [6], each "step" in this dependence is related to the contribution of one type of charge carrier, and its "height" and characteristic frequency enable the mobility and concentration to be determined. The method has been successfully applied to ice.

To conclude this description of the unusual electric properties of ice, we should mention the observation of negative currents of thermostimulated depolarization (TSD). TSD is normally observed in solids on heating samples precooled in a strong electric field. The TSD currents then naturally flow in the direction of decreasing the preliminary polarization. In ice, however, they sometimes flow in the opposite direction. This, at first sight, is as striking as if stretched and frozen rubber grew still longer on observation of negative TSD in ice can be explained by remembering that the motion of current carriers is determined not only by the force produced by the electric field, but also by the force produced by a certain configuration of $\mathrm{H}_{2} \mathrm{O}$ molecules formed by the current flow.

\section{Surface Physics of Ice}

The properties of the surface of ice also differ dramatically from those of a great number of solids. First, the ice surthin (10-200 $\AA$ ) quasi-liquid film that aids in rapid "sintering" of ice microcrystals into a monolithic mass. This film is largely responsible for sliding on ice and snow - or alternatively sticking.

Second, the ice surface possesses heating instead of growing shorter. The face is almost always covered with a

\section{Research Professor - Adjunct Professor} directing the education of students and scientists. in neutron diffraction work is also desirable. considered when fixing the individual allowance.

Further information can be obtained from:

Jens Als-Nielsen, Head, Physics Department, RNL

Applications should be filed with the: a very high electric conductivity, exceeding the bulk at $T>-20^{\circ} \mathrm{C}$.

Investigations of ice field transistors have prompted us to create a simple model of the ice surface that describes these two main properties [5]. We noticed that because of the difference in solubility of positive and negative ions in ice, bulk samples almost always turn out to be electrically charged. This charge is compensated by impurities present on the surface so that there is a potential difference of the order of several volts between the bulk and the surface. The potential gradient is established over a thin near-surface layer whose thickness is of the order of the screening length of the majority charge carriers. Electrostatic pressures, arising at the surface, are sufficient to "melt" the thin near-surface layer if the temperatures are not too low $\left(T>-20^{\circ} \mathrm{C}\right)$. Moreover, the growth of concentration of the charge carriers near the surface leads to an increase by several orders of magnitude in a stepwise manner at some critical value, as shown by Ryzhkin [8], thus transforming the ice to a superionic state and producing the high surface conductivity.

Comparing the experimentally measured thickness of quasi-liquid films with the surface conductivity yields the charge-carrier concentration and indi-

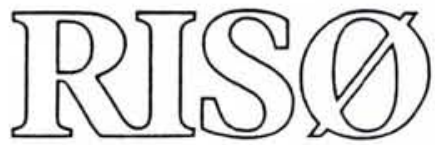

Risø National Laboratory (RNL) plans to appoint a research professor in experimental materials physics. The research professor should at the same time act as adjunct professor at the Faculty of Natural Sciences of the Copenhagen University.

The research professor will be employed in the Physics Department of RNL and is expected to direct the research performed by the group working on fundamental materials physics utilizing the experimental facilities at the Danish research reactor DR 3 . The position as adjunct professor is connected to the Physics Laboratory, H.C. Ørsted Institute, Copenhagen University and includes making contributions to the research projects of the laboratory and

At Risø, the materials physics research utilizing neutron diffraction comprises studies of magnetic materials, in particular phase transitions, at low temperatures, ceramic superconductors and polymer structures including biopolymers.

In collaboration with the Metallurgy Department at RNL, research is conducted which contributes to developing techniques for microstructural characterization, texture, and internal strain in metals, composites, and ceramic materials.

Applicants are expected to be well qualified in fundamental materials physics; experience

Applications in English will be submitted to an evaluation committee appointed jointly by the RNL Management and the Faculty of Natural Sciences. Tenure is restricted to five years but may be extended. The salary will be fixed according to agreement between the Ministry of Finance and the relevant negotiating organization. The merits of professorship will be

(Tel.: +4523712 12, Ext. 4711)

or from: Hans Henrik Andersen, Professor, Physics Laboratory,

H.C. Ørsted Institute, Copenhagen University (Tel.: +45 13531 33, Ext. 365).

Staff Office, Ris $ø$ National Laboratory, P.O. Box 49, DK-4000 Roskilde, Denmark,

to be received not later than the morning of 20 June, 1988. 


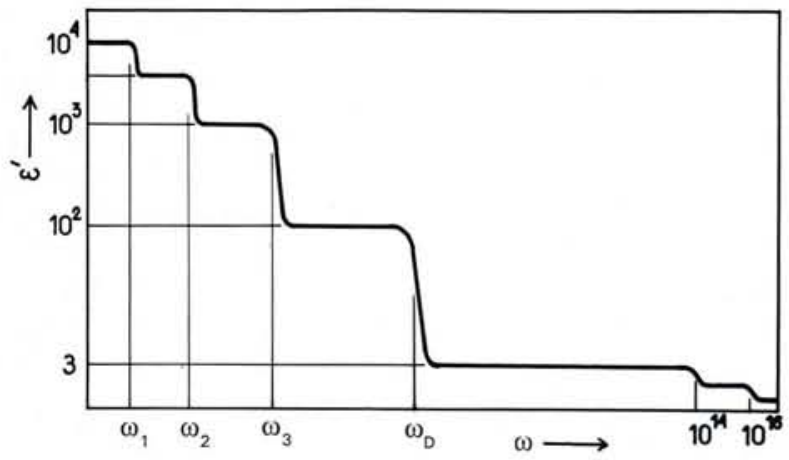

Fig. 7 - Frequency dependence of the real part of the dielectric permittivity of ice. "Steps" at $\omega_{1}, \omega_{2}, \omega_{3}$ and $\omega_{D}$ correspond to the motion of charge carriers of four different types.

cates the depth of the superionic surface layer. Fig. 8 illustrates the ice surface structure.

\section{Mechanical Properties of Ice}

In a number of natural phenomena, in which ice is subjected to alternating mechanical stresses - drift of temporary ice, compression of sea ice, etc., electromagnetic radiation is emitted. This has already found practical application, for example, in air reconnaissance of the regions of compression of sea ice for navigation and for forecasting the drift of temporary ice. However, until recently the nature of this radiation was enigmatic. Ice possessing a structure with a "statistical" (from the disordering of $\mathrm{H}_{2} \mathrm{O}$ molecules) symmetry centre cannot be piezoelectric and emit RF waves when vibrated.

In our experiments, ice subjected to non-uniform elastic stresses demonstrated a pseudo piezo effect. It turned out that proton charge carriers, deforming the ice lattice around themselves, produced a noticeable dilatation. In other words, ions $\mathrm{H}_{3} \mathrm{O}^{+}$and $\mathrm{OH}^{-}, \mathrm{L}$ and $\mathrm{D}$-defects occupy volumes different from those of $\mathrm{H}_{2} \mathrm{O}$ molecules. In the presence of elastic-stress gradients, the charge carriers move along them like an air bubble along a pressure gradient in water. Hence, in non-uniform fields of elastic stress, electric currents arise that can quite easily be measured, and they suffice to explain the RF emission of ice. In practice, ice becomes piezoelectric not only in the presence of temperature

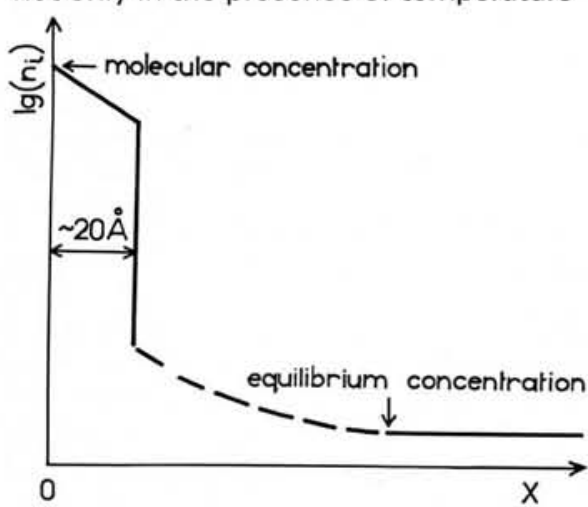

Fig. 8 - Distribution of charge carrier concentration near the ice surface. gradients but also impurity concentrations [9] which are common in natural conditions.

Dislocation currents, observed by Petrenko and Whitworth during plastic deformation of ice can now be attributed to the charge-carrier dilatation in ice. Charge carriers can be trapped by fields of non-uniform stresses existing around dislocations, the dislocations become electrically charged and by their motion generate electric currents. Carrier concentrations near such dislocation cores can exceed by several orders the mean values in the bulk. These must be taken into account in the description of the dislocation motion conditions, as shown by Petrenko and Ryzhkin.

Finally, we have solved rather accurately the problem of the reorientation of $\mathrm{H}_{2} \mathrm{O}$ molecules in ice under the action of elastic stresses, so creating a theory of inelastic relaxation of ice. One important outcome from this work has been a theory of propagation of sonic waves.

This short review of some recent results presents only a small part of the intensive studies in ice physics now being carried out across the world. We can expect this fascinating field of solid state physics to provide us with many important fundamental results which will find practical applications in future.

\section{REFERENCES}

[1] Jaccard C., Phys. Cond. Mat. 3 (1964) 99. [2] Hubbman M., Z. Phys. B 32 (1979) 127.

[3] Chesnakov V.A., Petrenko V.F., Ryzhkin I.A. and Zaretskii A.V., J. de Physique, C1 99 (1987).

[4] Petrenko V.F., Whitworth R.W. and Glen J.W., Phil. Mag. B 47 (1984) 259.

[5] Petrenko V.F., "Electrical Properties of Ice", VII International Symposium on the Physics and Chemistry of Ice, 1-5 September 1986, Grenoble.

[6] Petrenko V.F. and Ryzhkin I.A., Phys. Stat. Sol. (b) 121 (1984) 421.

[7] Petrenko V.F. and Maeno N., J. de Physique, C1 48 (1987) 115.

[8] Ryzhkin I.A., Sol. Stat. Com. 51 (1985) 57.

[9] Evtushenko A.A., Maeno N., Petrenko V.F. and Ryzhkin I.A., J. de Physique, C1 109 (1987).

\section{Hewlett-Packard Europhysics Prize 1989}

\section{Call for Nominations}

The Selection Committee for the Hewlett-Packard Europhysics Prize invites nominations for the 1989 award. The prize is given for an outstanding contribution to condensed matter physics within the previous five years, with the potential for leading to advances in the fields of electronic, electrical or materials engineering. Nominations may be submitted by EPS members as individuals or as representatives of a Division or Section.

During this decade the HewlettPackard Prize has been awarded to the following:

1980 - O. Krogh Andersen and

A.R. Miedema

1982 - K. von Klitzing

1983 - I. Silvera

1984 - G. Binnig and H. Rohrer

1985 - J. Als-Nielsen and M. Pepper

1986 - F. Mezei

1987 - I. Yanson

1988 - J.G. Bednorz and K.A. Müller

In order to maintain this extremely high standard, it is necessary that the Committee receive proposals which represent the breadth and strength of European condensed matter physics. It is also important that the submitted nominations be complete, and it is our experience that this is not always the case. They should comprise at least:

- a detailed motivation for the award,

including a clear definition of the work and its significance;

- a brief curriculum vitae of the nominee;

- a list of relevant publications.

It is also extremely helpful if we receive letters of support from authorities in the field, in which the importance of the work is evaluated. These can with advantage also be sollicited from nonEuropean physicists.

EPS members who know of a qualified candidate for the Hewlett-Packard Prize are urged to submit a complete nomination to the Selection Committee, to arrive before the end of August. All information will be treated as strictly confidential.

Nominations should be addressed to:

Selection Committee,

H-P Europhysics Prize

EPS, POB 69

$\mathrm{CH}-1213$ Petit-Lancy 2

\section{A.R. Mackintosh}

Chairman,

Hewlett-Packard Prize Selection Committee 